\title{
Sustentabilidade no Uso de Recursos Públicos Segundo a IFAC e o CIPFA: uma análise da Prática C de Governança Pública: definição de resultados em termos de benefícios econômicos, sociais e ambientais sustentáveis
}

\author{
Sustainability in the Use of Public Resources According to IFAC and \\ CIPFA: an analysis of public governance practice $C$ : definition of results \\ in terms of sustainable economic, social and environmental benefits
}

\author{
Patricia Gava Ribeiro ${ }^{1}$ \\ Gerson Ishikawa ${ }^{2}$ \\ Isaura Alberton de Lima ${ }^{1}$ \\ Paulo Nascimento Neto ${ }^{3}$ \\ Vanessa Ishikawa Rasoto ${ }^{1}$ \\ ${ }^{1}$ Universidade Tecnológica Federal do Paraná, Curitiba, PR, Brasil \\ 2Universidade Tecnológica Federal do Paraná, Ponta Grossa, PR, Brasil \\ ${ }^{3}$ Pontifícia Universidade Católica do Paraná, Curitiba, PR, Brasil
}

\begin{abstract}
Resumo
A Lei de Inovação estabeleceu a obrigatoriedade de criação de Núcleos de Inovação Tecnológica (NITs) nas Instituições Científicas, Tecnológicas e de Inovação (ICTs). Esses órgãos são responsáveis pela gestão da Propriedade Intelectual (PI) em tais instituições. Entre esses órgãos encontram-se as universidades públicas, o que justifica o interesse em examiná-las sob a ótica da governança pública, com o intuito de analisar especificamente a questão da sustentabilidade no uso dos recursos públicos disponíveis. Em vista disso, foi utilizado o relatório International Framework: Good Governance in the Public Sector, publicado em 2014 pela Federação Internacional de Contadores (IFAC) e pelo Instituto Chartered de Finanças Públicas e Contabilidade (CIPFA), tendo como foco a análise específica da prática C: Definição de resultados em termos de benefícios econômicos, sociais e ambientais sustentáveis. Após a análise de dados do questionário e das informações públicas vinculadas aos relatórios e aos sítios das instituições, das 30 universidades públicas participantes do estudo, infere-se que não há relação entre o indicador da Prática $\mathrm{C}$ e os resultados dos NITs, também se constatou a incipiência de tais órgãos no uso sustentável de recursos públicos.

Palavras-chave: Propriedade Intelectual. Governança Pública. Núcleo de Inovação Tecnológica.
\end{abstract}

\begin{abstract}
The Innovation Law established the obligation to create Technological Innovation Centers (NITs) in Scientific, Technological and Innovation Institutions (ICTs). Such bodies are responsible for the management of intellectual property (IP) in these institutions. Among these bodies are the public universities, what justify the interest in examining them from the perspective of public governance, seeking to analyze specifically the issue of the sustainability in the use of available public resources. In view of that, it was used the International Framework Report: Good Governance in the Public Sector, published in 2014 by the International Federation of Accountants (IFAC) and the Chartered
\end{abstract}


Institute of Public Finance and Accounting (CIPFA), focusing on the specific analysis of practice C: Defining results in terms of sustainable economic, social and environmental benefits. After the data analysis of the survey and the public information linked to the reports and websites of the institutions from the 30 public universities participating in the study, it is inferred that there is no relationship between the practice indicator $\mathrm{C}$ and the results of the NITs, as well as it was verified the incipience of such bodies in the sustainable use of public resources.

Keywords: Intellectual Property. Public Governance. Technological Innovation Center.

Área Tecnológica: Propriedade Intelectual. Governança Pública.

\section{Introdução}

Muito embora o Brasil possua atualmente um crescimento expressivo da produção científica (ORTIZ, 2018), o país permanece focado na produção de commodities (CASSIOLATO; SZAPIRO, 2015), apresentando dificuldades na transição do conhecimento para a inovação. Consequentemente, o Brasil possui uma quantidade limitada de patentes (MARINHO; CORRÊA, 2016; ORTIZ, 2018), sendo que muitas dessas patentes não são depositadas por residentes, demonstrando que elas são geradas no exterior, buscando proteção no mercado brasileiro (LOTUFO, 2009).

Nesse sentido, a Propriedade Intelectual (PI) possui um papel de destaque, pois atua como um mecanismo de disseminação do saber científico, além de ser um instrumento fundamental de proteção às criações intelectuais, possibilitando, por conseguinte, a geração de desenvolvimento econômico, nos casos em que os bens intangíveis protegidos se tornam uma inovação.

Para que o capital intelectual se torne inovação, é necessário que a tecnologia chegue ao setor produtivo (e posteriormente à sociedade) por meio da Transferência de Tecnologia (TT), sendo necessário haver sinergia entre a academia e as indústrias e/ou empresas.

Ao governo, com o propósito de uma redução na lacuna tecnológica brasileira em relação aos países desenvolvidos, cabe o papel de promotor dessa interação por meio, principalmente, da legislação. Prova disso são a Lei de Inovação (Lei n. 10.973/2004) e o recente Marco Legal da Ciência, Tecnologia e Inovação (Lei n. 13.243/2016), posteriormente regulamentado pelo Decreto n. 9.283/2018. Tais legislações, além de favorecerem a inovação, são responsáveis pela criação e pelo estabelecimento de competências dos denominados Núcleos de Inovação Tecnológica (NITs), estruturas responsáveis por realizar a ponte universidade-setor produtivo. Esses órgãos são encarregados tanto da gestão de PI nas Instituições Científicas, Tecnológicas e de Inovação (ICTs) quanto por realizar a transferência de tecnologia proveniente do saber acadêmico ao setor produtivo, gerando, assim, inovações que beneficiam a sociedade.

Consequentemente, as instituições públicas vêm buscando ganhar maior eficiência na gestão dos recursos públicos sob sua responsabilidade, utilizando mecanismos da governança pública com o intuito de obter maior economicidade e sustentabilidade no uso de tais recursos. Considerando que as instituições analisadas no presente estudo são universidades públicas, conhecer a forma como os NITs com tal vínculo institucional atuam em termos de gestão dos recursos públicos direcionados à proteção da PI e a consequente transferência de tecnologia é bastante relevante para se avaliar a solidez da administração pública.

Para tanto, utilizou-se como base o relatório International Framework: Good Governance in the Public Sector, publicado em 2014 pela International Federation of Accountants (a Federação 
Internacional de Contadores - IFAC) e pelo Chartered Institute of Public Finance and Accountancy (Instituto Chartered de Finanças Públicas e Contabilidade - CIPFA). Essas renomadas instituições estabeleceram no supramencionado relatório sete práticas de governança que podem ser aplicadas às instituições e que têm como intuito atingir os melhores resultados possíveis de maneira sustentável, prevalecendo sempre o interesse público (IFAC; CIPFA, 2014). Salienta-se que o presente estudo analisará exclusivamente a prática C: Definição de resultados em termos de benefícios econômicos, sociais e ambientais sustentáveis, fazendo parte de uma pesquisa mais ampla na qual foram investigadas todas as sete práticas.

\section{Referencial Teórico}

Para uma melhor compreensão da literatura que presta suporte ao presente estudo, esta seção é composta de três subseções, sendo que a primeira aborda os modelos de hélices, a subsequente versa sobre a transferência de tecnologia e a última discorre sobre a prática $\mathrm{C} d a$ governança pública.

\subsection{Os Modelos de Hélices}

De acordo com o modelo de Tríplice Hélice, proposto por Etzkowitz e Leydesdorff (1995), a sinergia entre governo, universidade e empresa, operando num processo de inovação em espiral, em que um agente assume o papel do outro, é fundamental para o crescimento econômico $e$ o desenvolvimento social na atual sociedade do conhecimento.

Outros autores sugerem ainda a existência de uma quarta hélice, incluindo a sociedade civil, e de um modelo de "Hélice Quíntupla", em que os ambientes naturais da sociedade atuam como a quinta hélice, destacando-se que o meio ambiente age como estímulo ao progresso dos sistemas de produção e inovação de conhecimento (CARAYANNIS; CAMPBELL, 2012).

À universidade, destina-se atualmente papel fundamental nesse contexto, ultrapassando a atribuição de formadora de recursos humanos qualificados e de responsável por atividades de pesquisa e extensão, para realizar sua "terceira missão", atuando de maneira estreita com o setor produtivo. Sob essa perspectiva, as universidades vêm sendo estimuladas pelo governo brasileiro a agregarem um papel mais proativo no tocante ao desenvolvimento econômico, social e tecnológico do país (OLIVEIRA; GIROLETTI, 2016).

\subsection{A Transferência de Tecnologia}

De acordo com Ortiz (2018), atualmente a pesquisa no Brasil é realizada sobretudo nas universidades públicas, sendo o Estado o maior promotor e financiador da ciência no país. Por conseguinte, muitas vezes, tais instituições são questionadas sobre a necessidade de proteção da propriedade intelectual, considerando que seu custeio se dá por meio de recursos provenientes da sociedade, o que leva a crer que os resultados de pesquisa deveriam ser disponibilizados para livre acesso. Contudo, há que se ponderar que frequentemente as tecnologias desenvolvidas se encontram ainda em fase embrionária, requerendo tempo e investimento até se tornarem inovações (LOTUFO, 2009). 
Nesse contexto, se revela a importância da transferência de tecnologia, pois é por meio dessa atividade que a complementaridade do desenvolvimento da tecnologia é repassada às empresas. Vale ressaltar que, segundo Sherwood (1992), a não realização de transferência de tecnologia pode ocasionar a perda de oportunidade de se obter reconhecimento público da produção de resultados concretos por parte da universidade e do possível aproveitamento de tal conhecimento pela sociedade.

A transferência de tecnologia pode se dar de diversas formas: consultorias, prestação de serviços, geração de novas empresas (spin-offs), pesquisas conjuntas e licenciamento de patentes (GARNICA; TORKOMIAN, 2005). Para o presente estudo interessam os licenciamentos, que são os contratos em que o titular (ICTs, neste caso específico, universidade) cede à outra parte (empresa) o direito de comercialização do conhecimento, mediante o pagamento de royalties. Nessa perspectiva, destaca-se que o NIT possui papel fundamental, estimulando a disseminação tecnológica enquanto assegura à universidade o custeio de despesas relativas à Pesquisa e Desenvolvimento (P\&D) por meio de royalties e taxas de licenciamento (SIEGEL; WALDMAN; LINK; 2003).

A inter-relação universidade-empresa é de "ganha-ganha", considerando que as empresas são favorecidas com produtos e processos de melhor qualidade provenientes do conhecimento científico, o que as torna mais competitivas no mercado. Sob outra perspectiva, as universidades, por meio da transferência de tecnologia, convertem $P \& D$ realizada em bens tangíveis, oportunizando retorno financeiro para o criador e para a instituição, possibilitando a utilização de tais recursos na própria universidade. Ademais, nesse processo, a sociedade como um todo é beneficiada por meio das inovações geradas, conforme é possível visualizar na Figura 1:

Figura 1 - Ciclo Virtuoso do Sistema de Propriedade Intelectual

CRIADOR

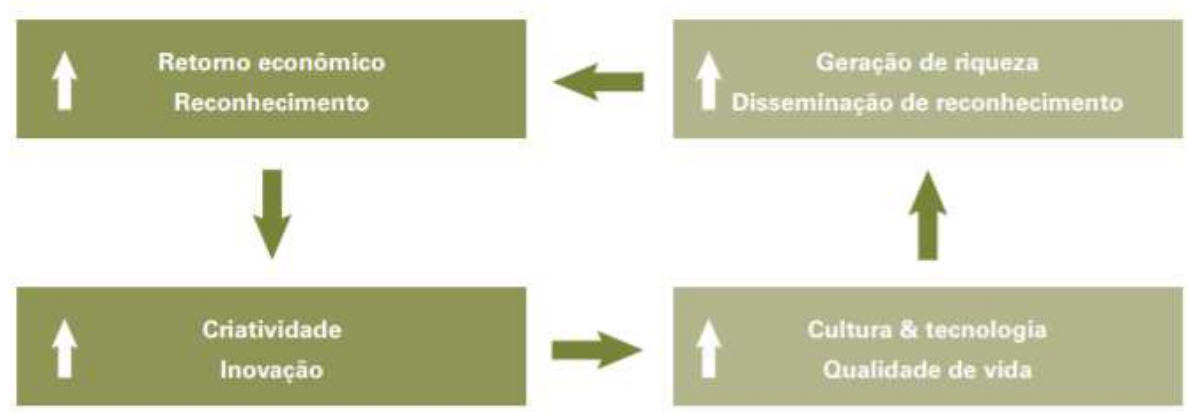

Fonte: Jungmann e Bonetti (2010, p. 18)

\subsection{A Prática C da Governança Pública}

Paralelamente, a governança pública vem sendo aplicada com o intuito de obtenção de maior eficiência na gestão dos recursos públicos, buscando reduzir custos e ampliar o nível de transparência das instituições. Oportunamente, pode-se enunciar que "[...] a governança é um processo dinâmico através do qual se dá o desenvolvimento político, através do qual a sociedade civil, o estado e o governo organizam e gerem a vida pública" (BRESSER-PEREIRA; 2001, p. 8). 
Já em relação às práticas de governança pública estabelecidas pela IFAC e pelo CIPFA, destaca-se a prática $\mathrm{C}$ - objeto do presente estudo - que pode ser observada na Figura 2:

Figura 2 - Práticas de governança pública, com destaque para a prática a ser analisada: $\mathrm{C}$ - Definição de resultados em termos de benefícios econômicos, sociais e ambientais sustentáveis

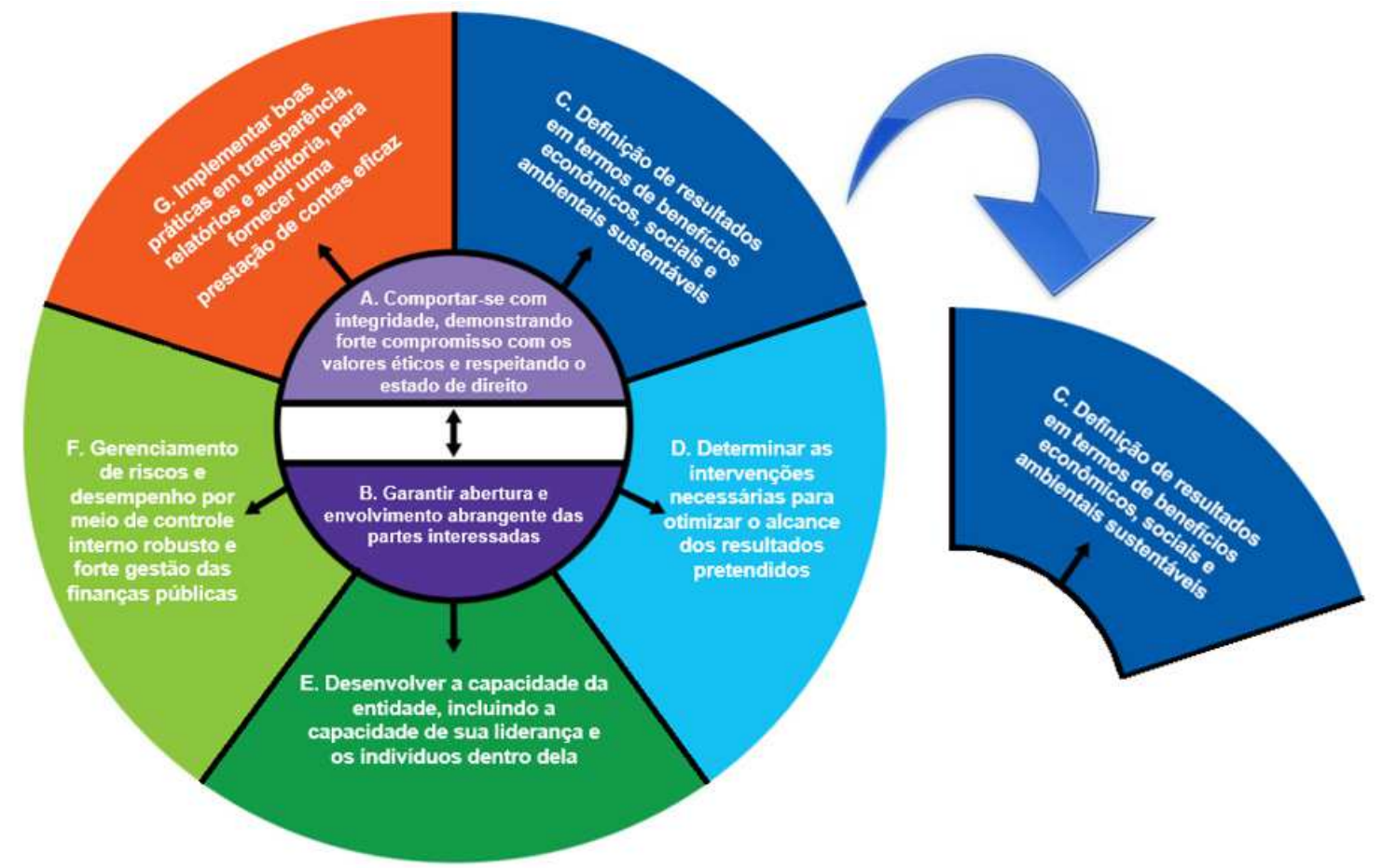

Fonte: Adaptada de IFAC e CIPFA (2014)

Tal prática sugere que a Administração Pública deve definir e planejar seus resultados de forma sustentável, assegurando que as decisões estejam dentro dos limites de autoridade e recursos. De acordo com os dois órgãos, sustentabilidade

[...] é a capacidade de uma entidade individual, comunidade ou população global de continuar a sobreviver com sucesso ao atingir seus resultados pretendidos (econômicos, ambientais e sociais) enquanto vive dentro de seus limites de recursos ${ }^{1}$. (IFAC; CIPFA, 2014, p. 20, tradução nossa)

Pode-se inferir que a sustentabilidade está fundamentada sobre três pilares estruturais: social, econômico e ambiental. Nesse sentido, é fundamental que todos os stakeholders participem do processo. Outrossim, é necessário que haja uma declaração formal por parte da instituição, incluindo os objetivos e os resultados almejados, tendo sempre como foco o interesse público em todos os momentos. Por isso, para que o objetivo almejado pudesse ser atingido, isto é, ponderar a extensão da Prática C nos NITs, todos esses aspectos se mostraram relevantes durante a elaboração do questionário.

\footnotetext{
1 "Sustainability is the capacity of an individual entity, community, or global population to continue to survive successfully in meeting its intended (economic, environmental, and social) outcomes while living within its resource limits".
} 


\section{Metodologia}

Inicialmente, a pesquisa compreendeu a análise de uma das sete práticas de governança pública preconizadas pela IFAC e pelo CIPFA no relatório denominado International Framework: Good Governance in the Public Sector, publicado em 2014. Essa é a prática C: Definição de resultados em termos de benefícios econômicos, sociais e ambientais sustentáveis.

Para aplicação da pesquisa foram selecionadas as universidades públicas das Regiões Sul e Sudeste que responderam ao Formulário para Informações sobre a Política de Propriedade Intelectual das Instituições Científicas, Tecnológicas e de Inovação do Brasil (FORMICT), elaborado pelo Ministério da Ciência, Tecnologia, Inovações e Comunicações (MCTIC), no ano de 2017 (com base nos dados de 2016). Justifica-se a escolha primeiramente em relação às universidades por serem instituições cujas atividades-fim estão voltadas ao ensino, à pesquisa e à extensão em todas as áreas do conhecimento humano (BRASIL, 2018a). Públicas para que fosse possível empregar práticas de governança pública.

Quanto às Regiões Sul e Sudeste, a escolha se deu pelo fato de que as instituições com maior volume de pedidos de patentes são universidades federais e estaduais dessas regiões - Instituto Nacional da Propriedade Industrial (INPI, 2018). Ademais, em relação especificamente aos pedidos de patente de invenção por estado de origem do depositante residente, no ano de 2017, verificou-se que do ranking de 10 estados, os seis primeiros estão localizados nas Regiões Sul e Sudeste (INPI, 2018). Além disso, segundo estudos, tais regiões concentram volume expressivo de práticas inovativas, resultando numa atividade econômica mais variada (GÓIS SOBRINHO; AZZONI, 2016; RODRIGUEZ; GONÇALVES, 2016).

Dessa forma, a população pesquisada totalizou 42 universidades públicas, considerando que uma foi excluída da pesquisa por se encontrar em processo de reestruturação de equipe e outra foi suprimida pelo fato de ter se estruturado muito recentemente e ter respondido a tão somente $50 \%$ das questões propostas (Figura 3). Desse total, 30 instituições responderam efetivamente à pesquisa.

Em relação à caracterização da pesquisa, essa possui natureza aplicada, pois pesquisas assim classificadas normalmente ocorrem nos casos em que o pesquisador busca obter mais informações a respeito de sua área de estudo (SEVERINO, 2007). Quanto ao objetivo, pode ser classificado como exploratório-descritivo, visando, além do que foi mencionado, à descrição pormenorizada de um certo fenômeno (MARCONI; LAKATOS, 2017). Assim sendo, foi utilizado como instrumento de coleta de dados o questionário, além de pesquisa documental junto aos portais institucionais. No que diz respeito à análise de dados, serão ponderadas as respostas das questões propostas, tanto em relação aos preceitos estabelecidos pela IFAC e pelo CIPFA (2014), quanto na bibliografia disponível sobre o tema. Outrossim, a partir da tabulação de dados, foi aplicado o Coeficiente de Correlação de Pearson, a fim de mensurar o nível de associação entre duas variáveis (no caso entre resultados de PI apresentados pelo NIT, considerando o volume de pedidos de PI, PI concedidos e PI licenciados e a média referente às questões da Prática C e do levantamento nos portais institucionais). 
Figura 3 - Universidades públicas das regiões Sul e Sudeste

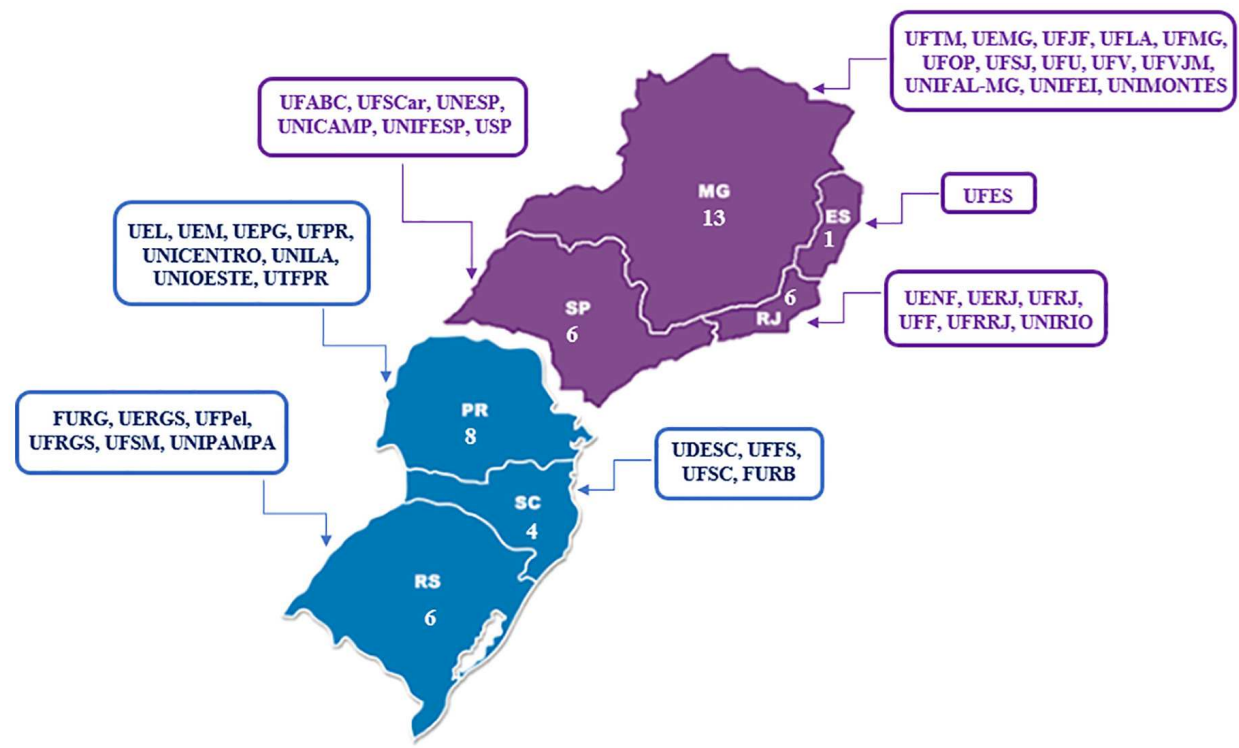

Fonte: Elaborada pelos autores deste artigo com base nos dados do Relatório FORMICT (BRASIL, 2017)

Os dados do Quadro 1 demonstram os critérios utilizados para análise das respostas fornecidas ao questionário elaborado, além do item pesquisado nos portais institucionais (pesquisa documental).

Quadro 1 - Critérios utilizados para análise das respostas - questionário/portais institucionais

\begin{tabular}{|c|c|c|c|}
\hline \multirow{6}{*}{ 造 } & QUESTÃO & FORMATO & CRITÉRIO UTILIZADO \\
\hline & 1 & $\begin{array}{l}\text { Múltipla escolha } \\
\text { com } 4 \text { opções } \\
\text { de resposta }\end{array}$ & $\begin{array}{l}\text { Definiu-se valor diferenciado para as alternativas, sendo: } \\
\text { - Sim, realiza análise e somente as que possuem } \\
\text { potencial de mercado são patenteadas (3); } \\
\text { - O NIT não realiza análise, mas solicita informações } \\
\text { sobre o potencial de mercado ao pesquisador (2); } \\
\text { - Embora seja realizada análise, todas as patentes com } \\
\text { critérios de patenteabilidade são patenteadas (1); } \\
\text { - Não é realizada análise e todas são patenteadas (0). } \\
\text { Por fim, dividiu-se por } 3 \text { para se obter a média. }\end{array}$ \\
\hline & 2 & $\begin{array}{l}\text { Múltipla escolha } \\
\text { com } 4 \text { opções } \\
\text { de resposta }\end{array}$ & $\begin{array}{c}\text { Definiu-se valor diferenciado para as alternativas, sendo: } \\
\text { - Sujeito à potencialidade de mercado da tecnologia (3); } \\
\text { - Pelos três primeiros anos (2); } \\
\text { - Outra periodicidade (especifique): (1); } \\
\text { - Todo o tempo de duração da proteção (0). } \\
\text { Por fim, dividiu-se por } 3 \text { para se obter a média. }\end{array}$ \\
\hline & 3 & $\begin{array}{l}\text { Escala Likert de } 5 \\
\text { pontos (2 escalas) }\end{array}$ & $\begin{array}{c}\text { - Notas de } 0 \text { a 4, para os } 5 \text { níveis de Escala Likert: Não se aplica } \\
\text { de modo algum (0); Pouco se aplica (1); Aplica-se razoavelmente } \\
\text { (2); Aplica-se bastante (3) e Aplica-se totalmente (4). } \\
\text { Total dividido por } 4 .\end{array}$ \\
\hline & 4 & $\begin{array}{l}\text { Múltipla escolha } \\
\text { com } 5 \text { opções } \\
\text { de resposta }\end{array}$ & $\begin{array}{c}\text { - Notas de } 0 \text { a } 5 \text {, considerando que quanto mais } \\
\text { meios, melhor (valor } 1 \text { para cada item). } \\
\text { Total dividido por } 5 \text {. }\end{array}$ \\
\hline & $\begin{array}{l}\text { Regimento } \\
\text { interno do } \\
\text { NIT (site) }\end{array}$ & $\begin{array}{l}\text { Atribuição de } \\
\text { notas } 0 \text { ou } 1\end{array}$ & $\begin{array}{l}\text { Definiu-se: } \\
\text { - Valor } 0 \text { para o caso de não constar no site e } \\
\text { - Valor 1,0 para o caso de constar no site. }\end{array}$ \\
\hline
\end{tabular}

Fonte: Elaborado pelos autores deste artigo 
Para análise dos resultados, foi utilizado o parâmetro estabelecido por Shimakura (2006) no qual a correlação, dentro de determinados intervalos, segue a seguinte classificação: de 0,00 a $0,19 \rightarrow$ bem fraca; de 0,20 a $0,39 \rightarrow$ fraca; de 0,40 a 0,69 $\rightarrow$ moderada; de 0,70 a 0,89 $\rightarrow$ forte e de 0,90 a $1,00 \rightarrow$ muito forte.

\section{Resultados e Discussão}

Na sequência, são apresentadas as questões propostas aos gestores de NITs contendo a sistematização da distribuição de frequências, de forma gráfica e tabular, e as discussões com base na literatura existente sobre o tema.

\section{Questão 1 - O NIT de sua instituição realiza uma análise de poten- cial de mercado em relação às patentes que atendam aos critérios de patenteabilidade?}

Antes de realizar a análise das respostas atribuídas a essa questão, faz-se necessário elucidar quais são os critérios de patenteabilidade. A fim de que seja concedida uma patente a uma invenção, são necessários três requisitos básicos (MACEDO; BARBOSA, 2000; OMPI/INPI, 2018): novidade, aplicação industrial e atividade inventiva. Quanto ao requisito novidade, este é considerado quando não houver conhecimento técnico contido no estado da técnica, compreendendo como estado da técnica a informação disponível ao público até a data de depósito da patente (MACEDO; BARBOSA, 2000). Há um conflito recorrente entre o patenteamento e a divulgação. Costumeiramente, as universidades e os institutos de pesquisa se interessam pela divulgação dos resultados de suas pesquisas. Entretanto, a divulgação ocorrida antes do pedido de patente ocasiona a eliminação do requisito novidade. Para driblar o conflito entre divulgação e patenteamento, há alguns dispositivos legais adotados em alguns países: a garantia de prioridade e o período de graça.

O primeiro recurso se dá por meio do depósito de uma breve descrição da invenção junto à instituição responsável pela proteção à propriedade intelectual. Já o segundo dispositivo requer o depósito de pedido de patente dentro de um período preestabelecido (MACEDO; BARBOSA, 2000). O período de graça corresponde aos 12 meses anteriores ao pedido. Nesse período, tanto o inventor quanto terceiros têm permissão para divulgar a invenção, podendo até mesmo apresentá-la em exposições internacionais oficiais (CAMPELLO; CENDÓN; KREMER, 2000).

O segundo requisito básico é o de aplicação industrial. A invenção deve ter por finalidade seu uso voltado à produção em escala industrial, compreendendo produtos e serviços (MACEDO; BARBOSA, 2000).

O terceiro requisito básico é o de atividade inventiva. Para atendimento a esse requisito, a invenção não pode decorrer de maneira óbvia do estado da técnica. "Assim, além da novidade em relação ao estado da técnica, a invenção deve envolver significativa criatividade em relação à técnica conhecida" (MACEDO; BARBOSA, 2000, p. 39). Essa avaliação deve ser realizada por um técnico no assunto (OMPI/INPI, 2018).

Nessa questão explicita-se que muito embora tenham sido obtidas respostas de $30 \mathrm{NITs}$, foram consideradas tão somente 29 , considerando que um NIT apresentou resposta contraditória, sendo, portanto, desconsiderada. 
Ao analisar o Gráfico 1, é possível verificar que a opção que foi mais assinalada é a de que o NIT não realiza análise, mas solicita informações sobre o potencial de mercado ao pesquisador (44,8\%). Em contrapartida, 37,9\% dos respondentes afirmaram que mesmo realizando análise, patenteiam todas as patentes que atendam aos critérios de patenteabilidade. Por outro lado, 13,8\% dos NITs afirmaram não realizar análise e que todas as patentes são patenteadas. De todos os NITs, somente um (correspondente a 3,5\% do total) informou que realiza análise e que apenas as patentes que possuem potencial de mercado são patenteadas.

Gráfico 1 - Análise de potencial de mercado em relação às patentes

Sim, realiza análise e somente as que possuem potencial de mercado são patenteadas

Embora seja realizada análise, todas as patentes com critérios de patenteabilidade são patenteadas

O NIT não realiza análise, mas solicita informações sobre o potencial de mercado ao pesquisador

Não é realizada análise e todas são patenteadas

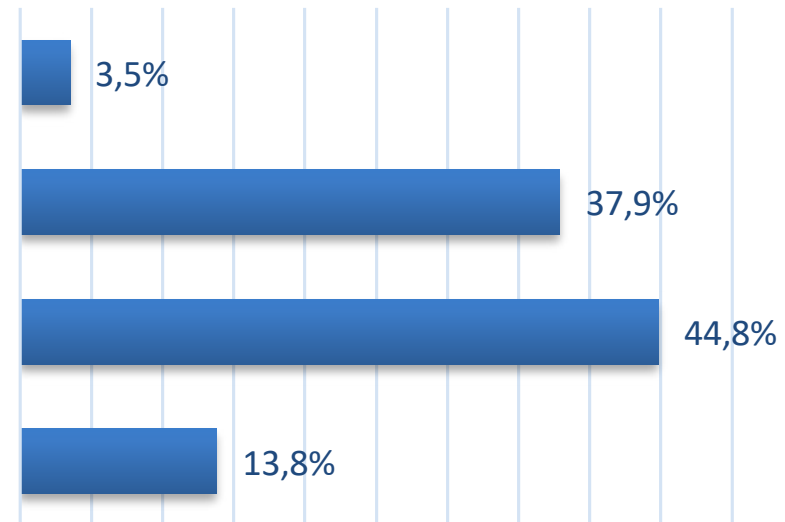

\begin{tabular}{|c|c|c|}
\hline ANÁLISE DE POTENCIAL DE MERCADO & RESPOSTAS & $\%$ \\
\hline $\begin{array}{l}\text { Sim, realiza análise e somente as que possuem } \\
\text { potencial de mercado são patenteadas }\end{array}$ & 1 & 3,5 \\
\hline $\begin{array}{l}\text { Embora seja realizada análise, todas as patentes com } \\
\text { critérios de patenteabilidade são patenteadas }\end{array}$ & 11 & 37,9 \\
\hline $\begin{array}{l}\text { O NIT não realiza análise, mas solicita informações } \\
\text { sobre o potencial de mercado ao pesquisador }\end{array}$ & 13 & 44,8 \\
\hline Não é realizada análise e todas são patenteadas & 4 & 13,8 \\
\hline Total & 29 & 100 \\
\hline Respondentes & \multicolumn{2}{|c|}{30} \\
\hline
\end{tabular}

Fonte: Dados obtidos pelos autores deste artigo com base nos resultados do questionário (2018)

Com base nos preceitos estabelecidos pela IFAC e CIPFA (2014), em que a Administração Pública deve aplicar os recursos públicos de maneira sustentável, considerando critérios como economicidade e eficiência, não passa despercebido o fato de que apenas uma instituição tenha respondido que realiza análise e que somente as patentes que possuem potencial de mercado são patenteadas.

\section{Questão 2 - No caso de não haver licenciamento da tecnologia disponível, por quanto tempo as anuidades/exames técnicos são mantidos pelo NIT?}

Do total de respondentes, $69,0 \%$ afirmaram manter as anuidades/exames técnicos no caso de não haver licenciamento da tecnologia disponível por todo o tempo de duração da proteção 
(Gráfico 2). Destaca-se que além dos cinco NITs que já consideram o potencial de mercado da tecnologia para estabelecer o prazo para manutenção de exames técnicos e anuidades (17,2\%), há outros dois que, apesar de terem informado manter as anuidades/exames técnicos por todo o tempo de duração da proteção, revelaram que: "está em análise a produção de uma normativa" e que "está alterando a sua Resolução para tratar da gestão das proteções que não tem potencial para licenciamento ou TT". Importante verificar que tais NITs manifestaram cuidado em relação à questão legal referente aos custos de manutenção de PI não licenciada (além dos que analisam o potencial de mercado). Infere-se aqui o despertar de um cuidado maior quanto à sustentabilidade no uso dos recursos públicos, conforme o que preconizam IFAC e CIPFA (2014).

Gráfico 2 - Não havendo licenciamento da tecnologia disponível, por quanto tempo as anuidades/ exames técnicos são mantidos pelo NIT

$$
\begin{array}{lllllllllllll}
0 \% & 10 \% & 20 \% & 30 \% & 40 \% & 50 \% & 60 \% & 70 \% & 80 \%
\end{array}
$$

Pelos três primeiros anos

Todo o tempo de duração da proteção

Sujeito à potencialidade de mercado da tecnologia

Outra peridiocidade

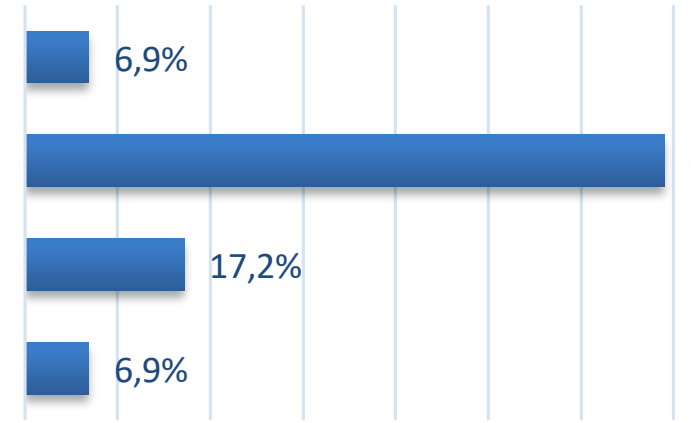

\begin{tabular}{|c|c|c|}
\hline PeRIODICIDADE & RESPOSTAS & $\mathbf{\%}$ \\
\hline Pelos três primeiros anos & 2 & 6,9 \\
\hline Todo o tempo de duração da proteção & 19 & 69,0 \\
\hline Sujeito à potencialidade de mercado da tecnologia & 5 & 17,2 \\
\hline Outra periodicidade & 2 & 6,9 \\
\hline Total & $\mathbf{2 8}$ & $\mathbf{1 0 0}$ \\
\hline Respondentes & \multicolumn{3}{|c|}{$\mathbf{3 0}$} \\
\hline
\end{tabular}

Fonte: Dados obtidos pelos autores deste artigo com base nos resultados do questionário (2018)

Ademais, 6,9\% dos NITs informaram manter as anuidades/exames técnicos pelos três primeiros anos, mesma porcentagem que afirmou considerar outra periodicidade: "ao menos 5 anos" e "durante todo o período de análise do INPI e por 2 anos após a concessão". Salienta-se que um dos NITs não respondeu a essa questão, enquanto outro não assinalou uma das respostas disponíveis, mas incluiu uma observação: "não temos nenhum caso e regulamentação para isso".

Muito embora deva ser considerado o uso eficiente dos recursos públicos, há que se considerar também que por vezes o mercado possa não estar ainda preparado para uma tecnologia protegida, sendo necessário ao NIT mantê-la pelo tempo necessário, assumindo os custos relativos a anuidades e exames técnicos. Denominado como Technology Readiness Level ${ }^{2}$ (TRL), os níveis de prontidão tecnológica são um método de análise para estimar a maturidade de uma

\footnotetext{
${ }^{2}$ TRL consiste num sistema de medição ordenado que possibilita avaliação da maturidade de uma tecnologia específica e a comparação robusta de maturidade entre diferentes tipos de tecnologia (MANKINS, 1995).
} 
tecnologia. De acordo com Velho et al. (2017, p. 121), "[...] a utilização crescente da ferramenta permite aos tomadores de decisão executar benchmarking, gerenciar riscos e deliberar sobre financiamento para prever quando lançar uma tecnologia ou produto no mercado".

\section{Questão 3 - No caso de não haver mais interesse na manutenção de uma propriedade intelectual protegida, quais os procedimentos adotados?}

Do total de respondentes (Gráfico 3), 30,0\% informaram que no caso de não haver mais interesse na manutenção de uma propriedade intelectual protegida, a desistência e o repasse desta ao inventor se aplicam bastante ou totalmente a seus NITs. Já para 6,7\% deles, aplica-se razoavelmente, enquanto a maior parcela, $60,0 \%$, declarou que pouco ou nada se aplica a seus respectivos NITs. Um dos NITs informou que "está em análise a produção de uma normativa".

Gráfico 3 - Procedimento adotado (desistência da patente e repasse ao inventor), no caso de não haver mais interesse na manutenção de uma propriedade intelectual

$$
0 \% \quad 10 \% \quad 20 \% \quad 30 \% \quad 40 \% \quad 50 \%
$$

Não se aplica de modo algum

Pouco se aplica

Aplica-se razoavelmente

Aplica-se bastante

Aplica-se totalmente

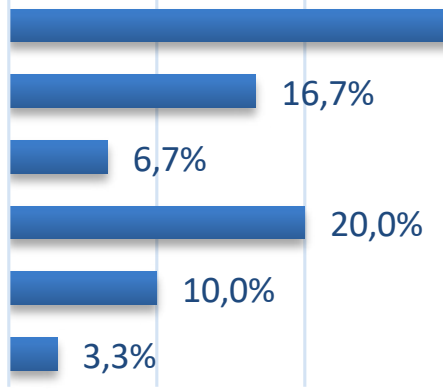

Outro

$3,3 \%$

\begin{tabular}{|c|c|c|}
\hline OPINIÃo & RESPOSTAS & $\mathbf{\%}$ \\
\hline Não se aplica de modo algum & 13 & 43,3 \\
\hline Pouco se aplica & 5 & 16,7 \\
\hline Aplica-se razoavelmente & 2 & 6,7 \\
\hline Aplica-se bastante & 6 & 20,0 \\
\hline Aplica-se totalmente & 3 & 10,0 \\
\hline Outro & 1 & 3,3 \\
\hline Total & $\mathbf{3 0}$ & $\mathbf{1 0 0}$ \\
\hline Respondentes & & $\mathbf{3 0}$ \\
\hline
\end{tabular}

Fonte: Dados obtidos pelos autores deste artigo com base nos resultados do questionário (2018) 
Gráfico 4 - Procedimento adotado (interrupção no pagamento de anuidades), no caso de não haver mais interesse na manutenção de uma propriedade intelectual

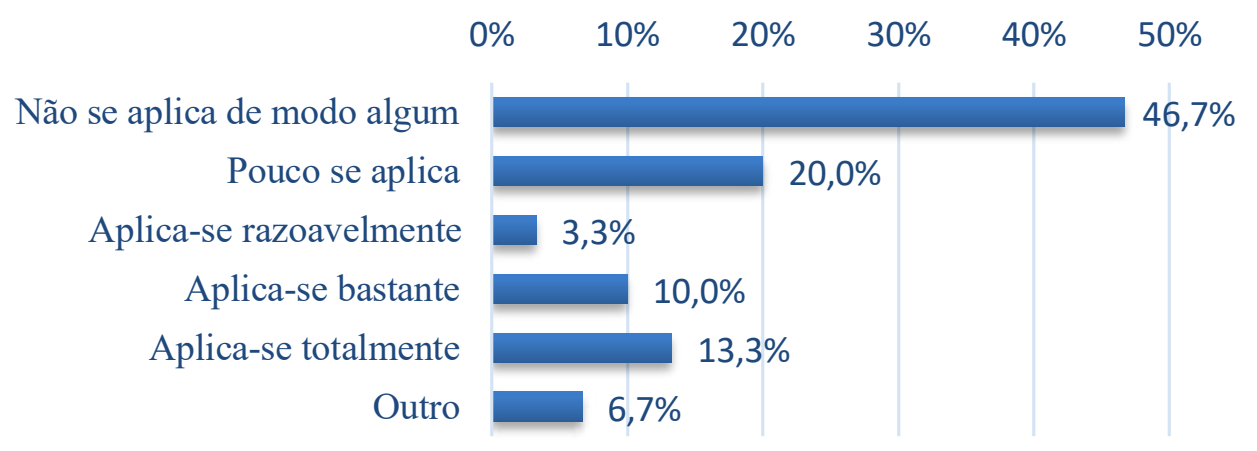

\begin{tabular}{|c|c|c|}
\hline OPINIÃo & RESPOSTAS & $\mathbf{\%}$ \\
\hline Não se aplica de modo algum & 14 & 46,7 \\
\hline Pouco se aplica & 6 & 20,0 \\
\hline Aplica-se razoavelmente & 1 & 3,3 \\
\hline Aplica-se bastante & 3 & 10,0 \\
\hline Aplica-se totalmente & 4 & 13,3 \\
\hline Outro & 2 & 6,7 \\
\hline Total & $\mathbf{3 0}$ & $\mathbf{1 0 0}$ \\
\hline Respondentes & & $\mathbf{3 0}$ \\
\hline
\end{tabular}

Fonte: Dados obtidos pelos autores deste artigo com base nos resultados do questionário (2018)

Já para a opção que considera, no caso de não haver mais interesse na manutenção

de uma propriedade intelectual protegida, que haja interrupção no pagamento de anuidades, $23,3 \%$ dos gestores informaram que se aplica bastante ou totalmente (Gráfico 4). Por outro lado, 3,3\% dos NITs declaram aplicar-se razoavelmente e, para 66,7\%, a interrupção pouco ou nada se aplica a seus respectivos NITs. Destaque para as respostas concedidas à opção "Outro", sendo que um dos respondentes informou que: "existe previsão legal para isso, contudo nunca foi aplicada" e outro afirmou que "o NIT está alterando a sua Resolução para tratar da gestão das proteções que não tem potencial para licenciamento ou TT".

Pela análise das duas respostas fornecidas pelos respondentes à opção "Outro", é possível verificar uma maior preocupação por parte dos NITs de universidades públicas com a eficiente aplicação dos recursos públicos, buscando resultados sustentáveis. Considerando que a proteção à propriedade intelectual requer significativa aplicação de recursos na obtenção e manutenção deste direito, faz-se necessário utilizar esse sistema de maneira responsável e sustentável (ORTIZ, 2018).

Importante destacar que a Lei de Inovação traz em seu texto orientação nesse sentido. No artigo 16, $\S 1^{\circ}$, inciso IV, consta como competência do NIT: "[...] opinar pela conveniência 
e promover a proteção das criações desenvolvidas na instituição” (BRASIL, 2004, art. 16). Portanto, cabe às universidades a análise quanto ao interesse na proteção de uma invenção, privilegiando o desenvolvimento tecnológico e social (ORTIZ, 2018).

Caso a instituição opte por não proteger determinada PI, essa propriedade intelectual se encontra amparada tanto pela Lei de Inovação, em seu artigo 11, quanto pelo Decreto n. 9.283/2018, em seu artigo 13. Dessa forma, ela poderá

[...] ceder seus direitos sobre a criação, mediante manifestação expressa e motivada, a título não-oneroso, nos casos e condições definidos em regulamento, para que o respectivo criador os exerça em seu próprio nome e sob sua inteira responsabilidade, nos termos da legislação pertinente. (ORTIZ, 2018, p. 113)

A decisão a respeito da cessão de direitos compete ao dirigente máximo da ICT pública, no prazo de seis meses, devendo ser ouvido o NIT (BRASIL, 2018b).

\section{Questão 4 - Com a intenção de estimular a geração de tecnologias sustentáveis, informações sobre os benefícios de programas como o "Patentes Verdes" (do INPI), são transmitidas de que forma?}

Em relação aos itens elencados aos respondentes a respeito do questionamento sobre o estímulo à geração de tecnologias sustentáveis, 36,7\% dos respondentes afirmaram que ocorre por meio de contato direto com membros da equipe do NIT, evidenciando haver um canal livre e de fácil acesso a esses órgãos, de acordo com a opinião dos gestores (Gráfico 5). O item palestras conta com $25,0 \%$ das respostas. Considerando os dois itens mais citados pelos respondentes, é possível constatar que, na percepção dos gestores de NITs, seu quadro de pessoal possui expressiva importância na transmissão de informações aos stakeholders.

$\mathrm{Na}$ sequência, os itens mais citados foram: site institucional $(13,3 \%)$ e as mídias sociais (10,0\%). Por outro lado, chama a atenção o fato de que seis instituições (10,0\%) afirmaram não informar sobre os benefícios de programas como o "Patentes Verdes" (do INPI). Nesse sentido, verifica-se que tais instituições estão desperdiçando a oportunidade de incentivar a criação de tecnologias sustentáveis, relacionadas ao meio ambiente (Hélice Quíntupla), evitando aderir a uma tendência global de priorização da análise de tecnologias verdes (MENEZES; SANTOS; BORTOLI, 2016).

Ademais, para a opção "informa por outro meio", foram obtidas as seguintes respostas (5,0\%): "formulário de solicitação de proteção"; "reuniões específicas e por segmento"; e "mailing list". 
Gráfico 5 - Transmissão de benefícios de programas como o "Patentes Verdes" (do INPI)

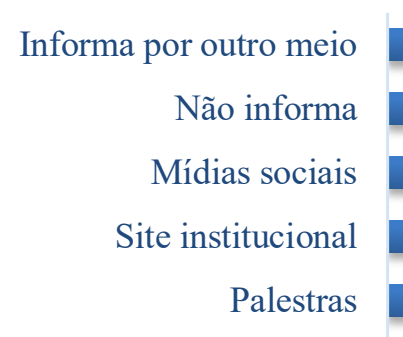

Contato direto com membros da equipe do NIT

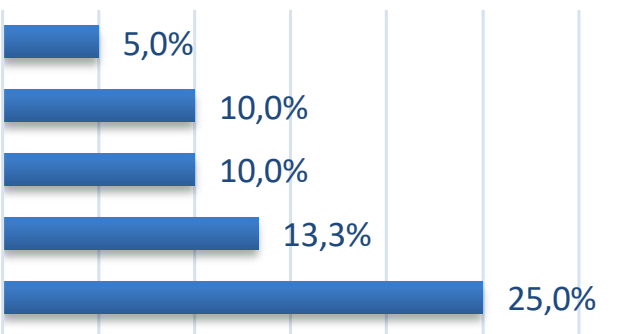

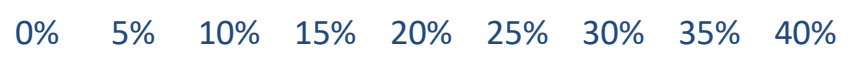

\begin{tabular}{|c|c|c|}
\hline MeIO UtILIzADo & RESPOSTAS & $\mathbf{\%}$ \\
\hline Informa por outro meio & 3 & 5,0 \\
\hline Não informa & 6 & 10,0 \\
\hline Mídias sociais & 6 & 10,0 \\
\hline Site institucional & 8 & 13,3 \\
\hline Palestras & 15 & 25,0 \\
\hline Contato direto com membros da equipe do NIT & 22 & 36,7 \\
\hline Total & $\mathbf{6 0}$ & $\mathbf{1 0 0}$ \\
\hline Respondentes & & $\mathbf{3 0}$ \\
\hline
\end{tabular}

Fonte: Dados obtidos pelos autores deste artigo com base nos resultados do questionário (2018)

Quanto ao item pesquisado nos portais institucionais dos NITs participantes do presente estudo, atendendo ao preconizado pela IFAC e pelo CIPFA (2014), de que as instituições devem apresentar uma declaração formal em que constem os objetivos a serem atingidos, foi possível verificar que dos 30 NITs, 21 apresentam tais declarações (comumente denominadas como Regimento Interno), ao passo que nove deles não apresentam tal documento. Muito embora o resultado tenha sido expressivo (70,0\% dos NITs), há a necessidade de que todos publiquem tal documento, considerando que esse deve ser utilizado para o planejamento em sua totalidade.

Ainda de acordo com Santos (2009, p. 87), a existência de uma política institucional clara e de fácil acesso à comunidade acadêmica confere legalidade e respaldo às ações desenvolvidas pelo NIT, uma vez que revela "[...] o grau de profissionalismo com que é tratado este tema e o compromisso institucional a respeito das atividades de desenvolvimento tecnológico".

Após a etapa de tabulação de dados foram utilizados os critérios para análise das respostas constantes no Quadro 1. Uma das variáveis considerada foi o somatório do número de pedidos de proteção à PI, quantidade de pedidos concedidos e volume de PI licenciada (dados informados no item de caracterização dos NITs no questionário aplicado), por universidade. A outra variável considerada foi definida pela média da adição das respostas atribuídas à prática de governança pública C (elencada pela IFAC e pelo CIPFA; 2014), à nota atribuída à presença ou ausência do regimento interno nos portais institucionais das universidades pesquisadas, por instituição. Após a sistematização das informações, foi possível verificar se havia uma possível correlação entre as variáveis, utilizando para tanto o Coeficiente de Correlação de Pearson (Tabela 1). 
Tabela 1 - Correlação entre resultado de PI e práticas de governança pública estabelecidas por IFAC e CIPFA (2014)

\begin{tabular}{|c|c|c|c|c|c|}
\hline NIT & $\begin{array}{l}\text { SOMA } \\
\text { PI }\end{array}$ & $\begin{array}{c}\text { MÉDIA PRÁTICA } \\
\text { C }\end{array}$ & NIT & SOMA PI & $\begin{array}{c}\text { MÉDIA } \\
\text { PRÁTICA C }\end{array}$ \\
\hline NIT 1 & 253 & 0,56 & NIT 16 & 236 & 0,47 \\
\hline NIT 2 & 305 & 0,36 & NIT 17 & 218 & 0,50 \\
\hline NIT 3 & 229 & 0,10 & NIT 18 & 123 & 0,24 \\
\hline NIT 4 & 109 & 0,17 & NIT 19 & 696 & 0,47 \\
\hline NIT 5 & 434 & 0,39 & NIT 20 & 59 & 0,71 \\
\hline NIT 6 & 790 & 0,48 & NIT 21 & 142 & 0,42 \\
\hline NIT 7 & 58 & 0,28 & NIT 22 & 528 & 0,53 \\
\hline NIT 8 & 132 & 0,29 & NIT 23 & 48 & 0,30 \\
\hline NIT 9 & 178 & 0,28 & NIT 24 & 2 & 0,04 \\
\hline NIT 10 & 150 & 0,34 & NIT 25 & 43 & 0,29 \\
\hline NIT 11 & 94 & 0,90 & NIT 26 & 1 & 0,56 \\
\hline NIT 12 & 297 & 0,56 & NIT 27 & 195 & 0,47 \\
\hline NIT 13 & 484 & 0,35 & NIT 28 & 1104 & 0,68 \\
\hline NIT 14 & 228 & 0,39 & NIT 29 & 458 & 0,31 \\
\hline NIT 15 & 652 & 0,07 & NIT 30 & 10 & 0,51 \\
\hline \multicolumn{6}{|c|}{ COEFICIENTE DE CORRELAÇÃO $(R)$ 0,15 } \\
\hline \multicolumn{6}{|c|}{ COEFICIENTE DE EXPLICAÇÃO $\left(R^{2}\right) 2,25 \%$} \\
\hline
\end{tabular}

Fonte: Elaborada pelos autores deste artigo com base no questionário aplicado e no levantamento efetuado nos portais institucionais

Analisando os dados contidos na Tabela 1, verifica-se que a correlação entre os resultados de PI apresentados pelos NITs (considerados como o somatório do número de pedidos de PI, quantidade de PI concedidos e volume de PI licenciada) e a média apresentada em relação à prática de governança pública C estabelecida pela IFAC e pelo CIPFA (2014), é considerada bem fraca $(r=0,15)$. Pelo valor apresentado é possível inferir que não há relação entre o indicador da Prática C e os resultados dos NITs.

Uma possível justificativa para tal resultado pode estar atrelada ao fato de que os NITs são bastante incipientes em termos de aplicação de práticas de governança e que, portanto, não realizam ainda uma gestão de PI com foco na sustentabilidade do uso dos recursos públicos disponíveis. Muito embora a Prática $\mathrm{C}$ se constitua num relevante item de governança, apresenta efeitos limitados nos resultados dos NITs. Nesse sentido, a avaliação dos impactos em termos de benefícios sustentáveis (econômicos, sociais e ambientais) permite maior transparência de gestão e visa melhorar a assertividade dos resultados dos projetos de inovação de forma sustentável; no entanto, aparentemente, como a governança dos NITs ainda é embrionária, os resultados de curto prazo e de maior visibilidade (ou seja, os econômicos) acabam se sobressaindo. 


\section{Conclusão}

Considerando a conjuntura brasileira atual em que o país apresenta elevado crescimento da produção científica, estrutura produtiva com foco na produção de commodities e quantidade incipiente de patentes, é inegável a importância da ação governamental no sentido de estimular a interação universidade-empresa. Para tanto, alguns dispositivos legais foram promulgados a partir do início da década passada, como a Lei de Inovação e o Marco Legal da Ciência, Tecnologia e Inovação.

Nesse cenário surge a figura do NIT, que, além de gerir a PI sob sua responsabilidade, atua como ponte entre o conhecimento científico gerado pela universidade e o setor produtivo por meio da transferência de tecnologia. Considerando que os NITs pesquisados estão inseridos em universidades públicas, desperta-se o interesse em verificar sob quais circunstâncias tem se dado sua atuação em termos de governança pública.

A governança pública tem sido aplicada nas instituições objetivando um uso mais sustentável dos recursos públicos, abrangendo a economicidade e a transparência da gestão junto aos stakeholders, pois, segundo Ortiz (2018, p. 106),

[...] uma série de estímulos, bem como de exigências às referidas Instituições, denotam uma preocupação com a propriedade intelectual na Universidade Pública e demonstram um tratamento mais atento e responsável com o patrimônio público intangível gerado nessa Instituição.

O presente estudo propôs então analisar uma das sete práticas elencadas no relatório International Framework: Good Governance in the Public Sector, publicado em 2014 pela IFAC e pelo CIPFA - a definição de resultados em termos de benefícios econômicos, sociais e ambientais sustentáveis. Assim, percebeu-se por meio da análise de dados a incipiência dos NITs em geral quanto ao uso consciente e sustentável de recursos públicos disponíveis. Ademais, constatou-se que os resultados em termos de volume de PI não podem ser explicados pela aplicação de práticas de governança pública nos NITs.

Para a consecução desse fim, foram propostos alguns questionamentos, bem como foi analisado se os NITs possuem em seus portais institucionais uma declaração formal contendo os objetivos do órgão. Como resultados, foi possível verificar que apenas um dos NITs realiza análise e que somente as patentes que possuem potencial de mercado são patenteadas. Em relação à manutenção das anuidades/exames técnicos no caso de não haver licenciamento da tecnologia disponível, aproximadamente $70 \%$ dos NITs afirmaram manter por todo o tempo de duração da proteção. Quanto à suposição de não haver mais interesse na manutenção de uma propriedade intelectual protegida, a desistência e repasse da mesma ao inventor pouco ou nada se aplica para $60 \%$ dos NITs e a interrupção no pagamento de anuidades pouco ou nada se aplica para mais de $66 \%$ dos NITs participantes da pesquisa. Em relação à publicização da declaração formal, dos 30 NITs, 21 o fazem em seus respectivos portais institucionais.

A despeito dos resultados apresentados, há que se considerar que surgiram algumas respostas que levam a crer que esteja ocorrendo um despertar da consciência por parte dos NITs de que necessitam realizar uma gestão de PI robusta, tomando decisões acerca da forma mais eficiente de gerir os bens intangíveis sob sua responsabilidade. Considerando que em geral os 
NITs são estruturas jovens, é natural que estejam ainda muito mais voltados às atividades de proteção da PI do que à atividade-fim de transferência de tecnologia. Por certo esse momento de transição mostra-se extremamente oportuno para a implementação de práticas de governança pública que visem o uso sustentável dos escassos recursos públicos à disposição.

Por fim, cabe fazer uma sugestão de estudos futuros, afinal de contas ficou muito evidente a ausência da análise de potencial de mercado de forma quase integral nos NITs, sendo que esse aspecto poderia ser melhor investigado sob o prisma da prática $\mathrm{C}$, especialmente atinente à viabilidade econômica inerente ao pagamento de anuidades das patentes pelas universidades e do impacto financeiro existente na manutenção dessas sem licenciamento.

\section{Referências}

BRASIL. Lei n. 10.973, de 2 de dezembro de 2004. Disponível em: http:/www.planalto.gov.br/ ccivil_03/_Ato2004-2006/2004/Lei/L10.973.htm. Acesso em: 10 dez. 2018.

BRASIL. Ministério da Ciência, Tecnologia, Inovações e Comunicações. Política de Propriedade Intelectual das Instituições Científicas, Tecnológicas e de Inovação do Brasil Relatório FORMICT 2016. Brasília: MCTIC, 2017. Disponível em: https://www.mctic.gov.br/mctic/export/ sites/institucional/tecnologia/propriedade_intelectual/arquivos/Relatorio-Formict-Ano-Base-2016.pdf. Acesso em: 19 abr. 2018.

BRASIL. Ministério da Educação. Qual é a diferença entre faculdades, centros universitários e universidades? [2018a]. Disponível em: http://portal.mec.gov.br/par/127-perguntasfrequentes-911936531/educacao-superior-399764090/116-qual-e-a-diferenca-entre-faculdadescentros-universitarios-e-universidades. Acesso em: 10 ago. 2018.

BRASIL. Decreto no 9.283, de 7 de fevereiro de 2018. [2018b]. Disponível em: http://www. planalto.gov.br/ccivil_03/_Ato2015-2018/2018/Decreto/D9283.htm. Acesso em: 10 fev. 2019.

BRESSER-PEREIRA, L. C. Uma nova gestão para um novo Estado: liberal, social e republicano. Revista do Serviço Público, [S.l.], v. 52, n. 1, p. 5-24, 2001.

CAMPELLO, B. S.; CENDÓN, B. V.; KREMER, J. M. Fontes de informação para pesquisadores e profissionais. Belo Horizonte: Ed. UFMG, 2000.

CARAYANNIS, E. G.; CAMPBELL, D. F. J. Mode 3 Knowledge Production in Quadruple Helix Innovation Systems. New York: Springer, 2012.

CASSIOLATO, J. E.; SZAPIRO, M. Os dilemas da política industrial e de inovação: os problemas da Região Sudeste são os do Brasil. In: CASSIOLATO, J. E.; SZAPIRO, M. Um olhar territorial para o desenvolvimento: Sudeste. Rio de Janeiro: BNDES, 2015. p. 284-317.

ETZKOWITZ, H.; LEYDESDORFF, L. The triple helix-university-industry-government relations: a laboratory for knowledge-based economic development. EASST Review, [S.l.], v. 14, n. 1, p. 1419, 1995.

GARNICA, L. A.; TORKOMIAN, A. L. V. Transferência de tecnologia universidade-empresa: fortalecimento de um modelo de cooperação através da propriedade intelectual. In: XII SIMPEP. São Paulo, 2005. Anais [...], Bauru, SP, 2005. 
GÓIS SOBRINHO, E. M.; AZZONI, C. R. Potencial inovativo da indústria nas regiões brasileiras. Revista Brasileira de Inovação, [S.l.], v. 15, n. 2, p. 275-304, jul.-dez. 2016.

IFAC; CIPFA. International framework: good governance in the public sector. [on-line]. IFAC; CIPFA, 2014. Disponível em: https://www.cipfa.org/policy-and-guidance/standards/internationalframework-good-governance-in-the-public-sector. Acesso em: 31 out. 2019.

INPI - INSTITUTO NACIONAL DA PROPRIEDADE INDUSTRIAL. Indicadores de Propriedade Industrial 2018: o uso do sistema de propriedade industrial no Brasil. Rio de Janeiro: INPI, 2018. Disponível em: http://www.inpi.gov.br/sobre/estatisticas/arquivos/pagina-inicial/indicadores-depropriedade-industrial-2018_versao_portal.pdf. Acesso em: 15 ago. 2018.

JUNGMANN, D. de M.; BONETTI, E. A. Inovação e propriedade intelectual: guia para o Docente. Brasília: SENAI, 2010.

LOTUFO, R. de A. A institucionalização de Núcleos de Inovação Tecnológica e a experiência da Inova Unicamp. In: LOTUFO, R. de A. Transferência de Tecnologia: estratégias para a estruturação e gestão de Núcleos de Inovação Tecnológica. Campinas, SP: Komedi, 2009. p. 41-73.

MACEDO, M. F. G.; BARBOSA, A. L. F. Patentes, Pesquisa \& Desenvolvimento: um manual de propriedade industrial. Rio de Janeiro: Fiocruz, 2000.

MANKINS, J. C. Technology Readiness Levels. White Paper, [S.l.], p. 5, April, 1995.

MARCONI, M. de A.; LAKATOS, E. M. Fundamentos de metodologia científica. 8. ed. São Paulo: Atlas, 2017.

MARINHO, B. C.; CORREAA, L. D. P. Novo marco legal da inovação no Brasil: breve análise dos reflexos das alterações na Lei n. 10.973 para os Núcleos de Inovação Tecnológica. Revista de Direito, Inovação, Propriedade Intelectual e Concorrência, [S.l.], v. 2, n. 1, p. 43-58, 2016.

MARTINEZ, C.; ZUNIGA, P. Contracting for technology transfer: Patent licensing and know-how in Brazil. Industry and Innovation, [S.l.], v. 65. p. 659-689, 2017.

MENEZES, C. C. N.; SANTOS, S. M. dos; BORTOLI, R. de. Mapeamento de Tecnologias Ambientais: um Estudo sobre Patentes Verdes no Brasil. Revista de Gestão Ambiental e Sustentabilidade, [S.I.], v. 5, n. 1, p. 18-32, 2016.

OLIVEIRA, M. do R. A.; GIROLETTI, D. A. Integração entre universidade e empresa: avaliação de projeto específico. Iberoamerican Journal of Industrial Engineering, [S.I.], v. 8, n. 16, p. 96-119, 2016.

OMPI - ORGANIZAÇÃO MUNDIAL DA PROPRIEDADE INTELECTUAL/INPI - INSTITUTO NACIONAL DA PROPRIEDADE INDUSTRIAL. Curso Geral de Propriedade Intelectual: DL101PBR, 2018.

ORTIZ, R. M. Os desafios da proteção da propriedade intelectual na universidade pública. PIDCC, [S.l.], v. 12, n. 2, p. 104-119, 2018.

RODRIGUEZ, R. S.; GONÇALVES, E. Hierarquia e concentração na distribuição regional brasileira de invenções por tipos de tecnologias. Rev. Bras. Inov., Campinas, SP, v. 16, n. 2, p. 225-266, 2016.

SANTOS, M. E. R. dos. Boas práticas de gestão em Núcleos de Inovação Tecnológica (NIT). In: SANTOS, M. E. R. dos. Transferência de Tecnologia: estratégias para a estruturação e gestão de Núcleos de Inovação Tecnológica. Campinas, SP: Komedi, 2009. p. 75-108. 
SEVERINO, A. J. Metodologia do Trabalho Científico. 23. rev. ed. São Paulo: Cortez, 2007.

SHERWOOD, R. M. Propriedade Intelectual e Desenvolvimento Econômico. São Paulo: Editora da Universidade de São Paulo, 1992.

SHIMAKURA, S. E. Atividades de Ensino. CE-003 Estatística II, 2006.

SIEGEL, D. S.; WALDMAN, D.; LINK, A. Assessing the impact of organizational practices on the relative productivity of university technology transfer offices: An exploratory study. Research Policy, [S.l.], v. 32, n. 1, p. 27-48, 2003.

VELHO, S. R. K. et al. Nível de Maturidade Tecnológica: uma sistemática para ordenar tecnologias.

Parcerias Estratégicas, [S.l.], v. 22, n. 45, p. 119-140, 2017.

\section{Sobre os Autores}

\section{Patricia Gava Ribeiro}

E-mail: patriciagava@utfpr.edu.br

Mestrado em Planejamento e Governança Pública pela Universidade Tecnológica Federal do Paraná em 2019.

Endereço profissional: Universidade Tecnológica Federal do Paraná, Reitoria, Avenida Silva Jardim, n. 775, Bloco V4, Rebouças, Curitiba, PR. CEP: 80.230-000.

\section{Gerson Ishikawa}

E-mail: gersonishikawa@utfpr.edu.br

Doutorado em Engenharia de Produção pela Universidade Federal de Santa Catarina em 2010.

Endereço profissional: Universidade Tecnológica Federal do Paraná, Câmpus Ponta Grossa, Rua Doutor Washington Subtil Chueire, n. 330, Jardim Carvalho, Ponta Grossa, PR. CEP: 84.017-220.

\section{Isaura Alberton de Lima}

E-mail: alberton@utfpr.edu.br

Doutorado em Engenharia de Produção pela Universidade Federal de Santa Catarina em 2004.

Endereço profissional: Universidade Tecnológica Federal do Paraná, Reitoria, Avenida Sete de Setembro, n. 3.165, Rebouças, Curitiba, PR. CEP: 80.230-901.

\section{Paulo Nascimento Neto}

E-mail: paulo.neto@pucpr.br

Doutorado em Gestão Urbana pela Pontifícia Universidade Católica do Paraná em 2015.

Endereço profissional: Pontifícia Universidade Católica do Paraná, Rua Imaculada Conceição, n. 1.155, Prado Velho, Curitiba, PR. CEP: 80.215-901.

\section{Vanessa Ishikawa Rasoto}

E-mail: ishikawa@utfpr.edu.br

Doutorado em Engenharia de Produção pela Universidade Federal de Santa Catarina em 2006.

Endereço profissional: Universidade Tecnológica Federal do Paraná, Reitoria, Avenida Sete de Setembro, n. 3.165, Rebouças, Curitiba, PR. CEP: 80.230-901. 